\title{
Studies on biomechanics of skeletal muscle based on the working mechanism of myosin motors: An overview
}

\author{
YIN YueHong*, GUO Zhao, CHEN Xing \& FAN YuanJie \\ State Key Laboratory of Mechanism System and Vibration, Institute of Robotics, Shanghai Jiao Tong University, Shanghai 200240, China
}

Received March 19, 2012; accepted May 23, 2012

\begin{abstract}
Skeletal muscle is the source of human body motion. Many scholars have been studying in this field to reveal its contraction mechanism, and relevant achievements have been awarded the Nobel Prize. This paper reviewed the current researches on biomechanics of skeletal muscle, and concluded two strategies (top-down and bottom-up methods) for the biomechanical research of skeletal muscle. Moreover, this paper generalized two major aspects of muscle research: (1) the multi-force coupling mechanism and the collective operation mechanism of molecular motors; (2) the bioelectrochemical driving and control principium of muscle contraction. We discussed the solution for experimental verification and induced a novel idea to study the biomechanics of skeletal muscle based on the microscopic working mechanism of molecular motor, which is the origin of muscle contraction. Finally we analyzed the disadvantages in existent researches and explored future directions that need further studies.
\end{abstract}

skeletal muscle, molecular motor, biomechanical model, sarcomere, working mechanism

Citation: Yin Y H, Guo Z, Chen X, et al. Studies on biomechanics of skeletal muscle based on the working mechanism of myosin motors: An overview. Chin Sci Bull, 2012, 57: 4533-4544, doi: 10.1007/s11434-012-5438-y

Skeletal muscles are connected to body joints through tendons and contract to generate limb movements under the control of nervous systems. In human body there are over 600 skeletal muscles, whose weight is $\sim 40 \%$ of the whole body. Due to its important role in limb motion, skeletal muscle has been extensively studied by global researchers since 1930 s, aiming at revealing the biomechanical rules of muscle contraction.

The earliest breakthroughs were made by Hill [1] and Huxley [2], both being Nobel Prize winners. Their achievements laid the foundation of the biomechanical study of skeletal muscle. Based on their works, later studies are mostly aimed at the structure and function of muscle $[3,4]$, respectively. Recently, with the rapid development of micro/nano technologies, skeletal muscle research has become a focus of biomechanics. The purpose of the researches is to build biomechanical models via investigating the mechanical properties of skeletal muscle, and to introduce the models to applications such as motion analysis of

*Corresponding author (email: yhyin@ @stu.edu.cn) human body, bionics, and rehabilitation engineering.

Skeletal muscle has active contraction and passive stretch characteristics on macroscopic level. Plenty of models have been proposed to describe such passive tensile properties [5], which are similar to that of other soft tissues. However, different from these tissues (tendon and ligament), skeletal muscle can actively contract under the control of nervous systems. This makes it a key point in the biomechanical research of skeletal muscle. Microscopically, the contraction of skeletal muscle involves procedures including neural system control, action potential (AP) propagation, $\mathrm{Ca}^{2+}$ diffusion, working of molecular motors and sarcomere contraction, and therefore the biomechanical study of skeletal muscle is inevitably complicated and difficult.

This paper summarized the microscopic studies on biomechanical principles of skeletal muscle in recent years. Combining relevant domestic and international works, we reviewed the progress of the biomechanical research from the following aspects: the anatomy structure of skeletal muscle, biomechanical models, microscopic mechanism of contraction and the bioelectrochemical driving and control 
principium. We discussed the deficiencies of existent works and the research directions requiring further exploration in the future.

\section{Anatomy structure of skeletal muscle}

The physiological structure of skeletal muscle is shown in Figure 1. From the macroscopic point of view, skeletal muscle is formed by muscle fibers composed of parallel myofibrils, and the fibers are surrounded by connective tissues, proprioceptors and motor neurons (Figure 1(a)). On mesoscopic level, myofibril consists of serial cylindrical units called sarcomeres, which are the fundamental units of skeletal muscle (Figure 1(b)). At microscopic scale, sacromere mainly contains thick and thin filaments. Thin filament is composed of actin, troponin (Tn) and tropomyosin (Tm) (Figure 1(c)), and myosin II motors form the thick filament (Figure 1(d)), which is connected to Z-lines through elastic titin [6]. Myosin II is one kind of linear molecular motor, and sarcomere contracts under the collective work of myosin motors toward thin filaments. In 1993, the molecular structure of myosin II is determined by Rayment with the X-ray diffraction techniques [7,8], which revealed that myosin II is composed of three parts: the head, neck and tail domains. The head domain accommodates the actin binding site and the ATP catalytic site, and ATP hydrolysis energy is utilized by myosin head to generate force against the thin filament. The majority of the neck domain is an $\alpha$-helix connected to myosin head, and the helix is bound with the essential and regulatory light chains, which regulate the head activities [9]. Holmes et al. [10] observed the three dimensional structure of myosin and the binding process of the acto-myosin system with scanning electron microscopy.

\section{Biomechanical model of skeletal muscle and its research strategy}

The biomechanical model of skeletal muscle is one of the fundamental researches in life science [11,12], and the most representative model was developed by Hill who deduced the Hill's equation via rapid release experiments of frog sartorius muscle in 1938 [1]. This equation describes the force-velocity relation of muscle, while it only represents one aspect of muscle characteristics. Aiming at complete description of macroscopic muscle features, Hill successively proposed the three-element model, which is constituted by a contractile element, a serial spring element and a parallel spring element. Based on in vitro muscle experiments, Hill's model demonstrates the macroscopic properties of skeletal muscle, while its biggest shortage comes from the low accuracy and over simplifications during modeling. Consequently, many researchers have made modifications to this model, e.g. Zajac et al. [13] revised the Hill's model by taking into account the effect of tendon on muscle force. The Hill-type model and its modifications laid the foundation for the biomechanical research of skeletal muscle and described the macroscopic mechanical features of muscle contraction; however, they neglected the microscopic characteristics due to technical reasons. Thus the models are not capable of effectively representing the mechanical properties of living muscle, leading to its disadvantages in real applications.

With the development of microscopy technologies, the micro-structures of skeletal muscle have been gradually revealed, enabling the biomechanical research to transfer from macroscopic scales to microscopic ones. According to the observation results, Huxley et al. [14,15] proposed the 'sliding filament theory', which explains muscle contraction with the relative sliding between thin and thick filaments. This theory has made great contributions to skeletal muscle research. After that, in order to further describe the microscopic mechanics of muscle, they proposed the cross-bridge model [16] and provided the binding rate equations for cross-bridges with several assumptions. The Huxley-type model is recognized as the significant basis for microscopic biomechanical research of skeletal muscle. In 1966, Gordon et al. [17] studied the force-length relation of a single muscle fiber and showed that muscle tension is proportional to the overlap length between the thick and thin filaments within a certain range, which means that the maximum tension occurs at the optimal length, while muscle force gradually approaches zero with continued stretch due to the decline of overlap length. Later, Zahalak et al. [18] revised

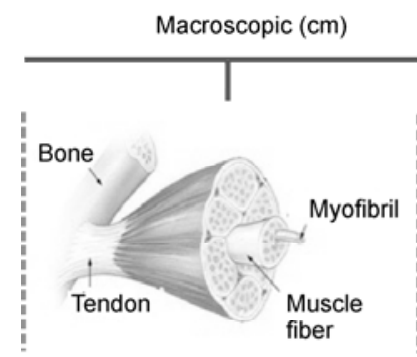

(a)

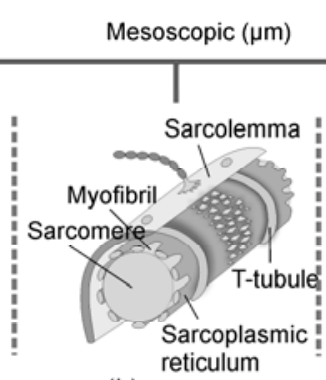

(b)

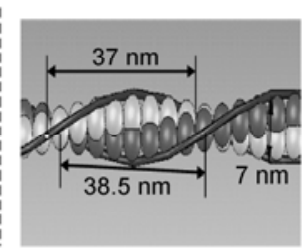

(c)

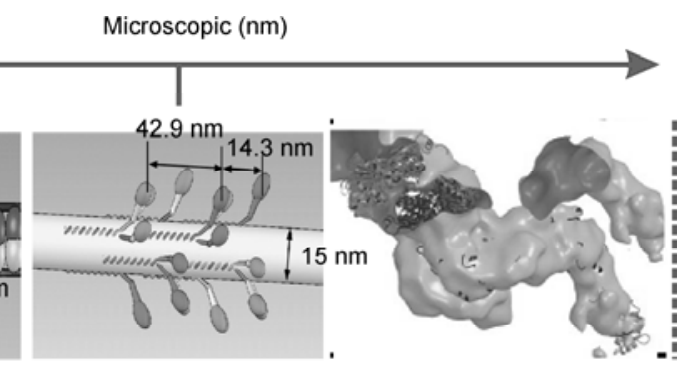

(d) (e)

Figure 1 The structure of skeletal muscle in each scale. (a) Skeletal muscle; (b) myofibril; (c) thin filament; (d) thick filament; (e) myosin head. 
the rate equations in the Huxley's model by introducing the $\mathrm{Ca}^{2+}$-activation process of actin filament. The Huxley's model and its modifications are based on microscopic muscle structures, and the models derived the force formula of skeletal muscle with the hypothesis of attachment-detachment cycles of acto-myosin system, while the correlation between microscopic mechanism and macroscopic features of muscle is absent. It is worth noting that Piazzesi et al. [19] measured the axial motions of myosin head via a new X-ray interference technique in 2002, and provided compelling support for the power stroke model rather than the attachment-detachment hypothesis proposed by Huxley.

Thus, while Huxley proposed the modeling method based on the microscopic mechanism of skeletal muscle, the description for the cycling process of myosin motors is insufficient, and the microscopic and macroscopic mechanical properties of muscle contraction have not been effectively unified. With the rapid development of nanotechnology, the molecular structures of skeletal muscle have been observed, and the interactions between myosin and thin filament have been measured with the applications of atomic force microscopy (AFM), optical tweezers/traps and other single molecule operation technologies [20]. These new methods have enabled in-depth analysis of the cyclic working mechanism of molecular motors, based on which the collective working mechanism of the motors can be studied with the non-equilibrium statistical mechanics. If the dynamical model of sacromere is established, a new biomechanical model of skeletal muscle can be built according to the serial and parallel features of sacromere structure. This is the bottom-up (from microscopic to macroscopic scale) strategy for biomechanical research of skeletal muscle.

There are mainly two methods for the verification of biomechanical models according to the existent researches. One is the inverse dynamical method of human body motion, i.e. obtaining the dynamic force of a single muscle with the body motion and joint force known. The mechanical information of body motion, such as the joint angle, velocity and force can be measured with instruments like the gait analysis system, and the torques of the joints can be inversely calculated via building a joint system model of human. Then the corresponding muscle force can be obtained with the constrained optimization method with the knowledge of muscle moment arms based on anatomy [21]. However, the problem of redundant muscle forces within the inverse dynamical equation has limited its application and generalization. The other method is the forward dynamics for human body movements. It has provided a new way for the calculation of muscle force by building mathematical models for the processes from neural signal generation to joint motion. Neptune et al. [22] analyzed human lower limb motion with 17 pieces of Hill-type virtual skeletal muscles based on the forward dynamical method. It is worth mentioning that Yang et al. [23] established a lower limb forward dynamical model which combined muscle- tendon mechanical features and the excitation-contraction coupling dynamics, while the accuracy of calculation outputs was limited due to the adoption of the classic Hill-type model.

It should be noted that difficulties always exist in the experimental verification of the biomechanical models of skeletal muscle. At present, the models are mostly based on hypotheses or in vitro experiments on animal muscle, whose properties are not guaranteed to be completely consistent with that of living muscle. However, it is definitely impossible to directly take experiments on human living muscle in vivo, while experimental verification is essential to the research of biomechanical models. Thus a natural question is whether this problem can be solved with other methods. The answer is positive for that the biomechanics of human body can be analyzed with equipments like electromyograph, motion capture system and force measuring platform, and combined with the knowledge of anatomy, the verification of the models can be done by obtaining the dynamic motion information of muscle. Surface electromyography (sEMG) is induced by the superposition of APs on muscle fibers, and because of the body motion procedures of neural signal-muscle contraction-joint movement, sEMG can reflect mechanical features of muscle such as the contraction force and speed. During joint motions, the activation levels of the involved muscles can be characterized by sEMG via attaching suitable electrodes to the skin.

Thereby, the motion and force information can be collected with corresponding equipments, and the validation data can be acquired by torque calculations via the inverse dynamics. On the other hand, we can build biomechanical models of skeletal muscle from the perspective of microscopic structures, calculate the joint torques by the forward dynamical analysis with the combination of sEMG and anatomy, and then verify the effectiveness of the models via comparing the resulted data with that generated by the inverse dynamics. This is the top-down strategy (from macroscopic to microscopic scale) on experimental research of skeletal muscle biomechanical model.

In summary, we can firstly establish the biomechanical models for skeletal muscle with the bottom-up method based on the microscopic research of dynamic process of muscle contraction, and then solve the problem of unifying the microscopic mechanics and the macroscopic mechanical model with the top-down experimental verifications. This is an effective way for the biomechanical research of skeletal muscle. In order to establish the new biomechanical model, it is critical to study the microscopic mechanism of muscle contraction and the bioelectrochemical driving and control principium of skeletal muscle; therefore, we need to analyze the multi-force coupling mechanism of the approaching and attaching process between myosin and actin, as well as the working cycles of myosin motor. It is also essential to study the collective properties of molecular motors and the macroscopic mechanical features of the serial and parallel sar- 
comere structures.

\section{Microscopic mechanism of skeletal muscle contraction}

As mentioned above, muscle contraction is produced by the collective working of myosin motors toward the thin filament. Molecular motor is a kind of enzyme molecular machine that converts chemical energy directly into mechanical work, and it generates linear or rotary motions fuelled by ATP hydrolysis energy that widely serves life activities [24]. Muscle force comes from the interaction between myosin and actin. Firstly the APs on muscle fibers trigger the open of $\mathrm{Ca}^{2+}$ channels on sarcoplasmic reticulum (SR), resulting in the release of $\mathrm{Ca}^{2+}$ into cytoplasm and the rise of $\left[\mathrm{Ca}^{2+}\right]$, successively $\mathrm{Ca}^{2+}$ binds to Tn and causes a conformational change of Tm, unblocking the binding sites for myosin [25]. Then the power stroke of myosin starts through its binding with the actin filament and the hydrolysis of ATP [26], and this process is referred to as the Lymn-Taylor cycle [27]. The collective working of myosin motors generates the relative sliding between thick and thin filaments. The study on the microscopic mechanism of muscle contraction mainly includes the following aspects: (1) the force field analysis of the myosin working environment, aiming at elucidating the working mechanism of molecular motors under multi-force coupling interaction; (2) the explanation for the working cycle of a single molecular motor from the perspectives of the conformational change mechanism and the Brownian ratchet mechanism; (3) the study on the collective behavior of myosin motors, for the purpose of providing the transition between microscopic and macroscopic-scale mechanisms for muscle contraction, as well as helping with the establishment of biomechanical models of skeletal muscle.

\subsection{Multi-force coupling mechanism for molecular motors}

Existent researches on the microscopic mechanism of muscle contraction are mainly focused on the status analysis of myosin motors, while the dynamic binding process between myosin and actin (especially the force generation mechanism and the dynamical behavior) is seldom covered. According to interaction distance between myosin and actin, the forces during the approaching process may include electrostatic force, van der Waals and Casimir forces, solvent force, and each force has its own effective condition and range [28]. Casimir force is similar to van der Waals force. In vacuum, Casimir effect dominates when the separation between the particles is $5 \mathrm{~nm}-1 \mu \mathrm{m}$, while in situations where the distance is less than $5 \mathrm{~nm}$, van der Waals force becomes significant, although this distance may be shorter in solvent. Recently, a repulsive Casimir force has been identified and measured in a fluid by Federico et al. [29], and the results showed that the force can suspend particles in the solvent. The verification of Casimir force has provided us strong support for taking it into account in the analysis of multi-force coupling mechanism. Compared with Casimir force, van der Waals force is a shorter-ranged molecular interaction, thus for large proteins like myosin and actin, van der Waals force also plays a crucial role in their interactions. On the other hand, X-ray crystallography studies on the structure of the acto-myosin system have shown that myosin head has positively charged segments and the binding sites on actin are negatively charged, indicating that electrostatic interactions facilitate the binding process [7,8]. Liu et al. [30] concluded that electrostatic force is dominant as being 3-4 folds larger than van der Waals force via molecular dynamics simulations (MDS) of the interaction between myosin and actin.

Meanwhile, new progresses have been made on the interaction force and displacement measurements of the acto-myosin system. The most representative work was done in 1993 by Finer et al. [20]. They measured the force produced by myosin and its walking distance alone the thin filament with optical tweezers for the first time. It was shown that myosin could move $5-10 \mathrm{~nm}$ and the force is 4-6 pN during one ATP hydrolysis cycle, as shown in Figure 2(a). Nakajima et al. [31] measured the interaction force during the binding process of myosin and actin with AFM, and found it to be within the range of $18.4-24.7 \mathrm{pN}$, as shown in Figure 2(b).

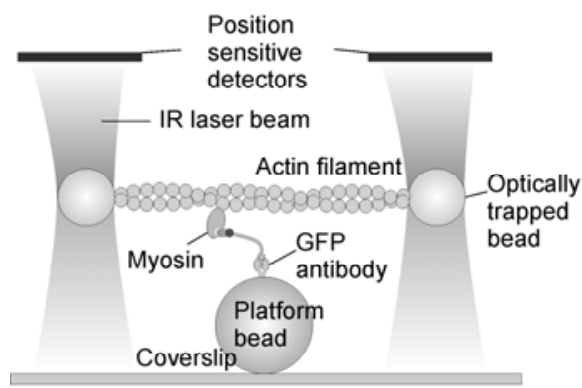

(a)
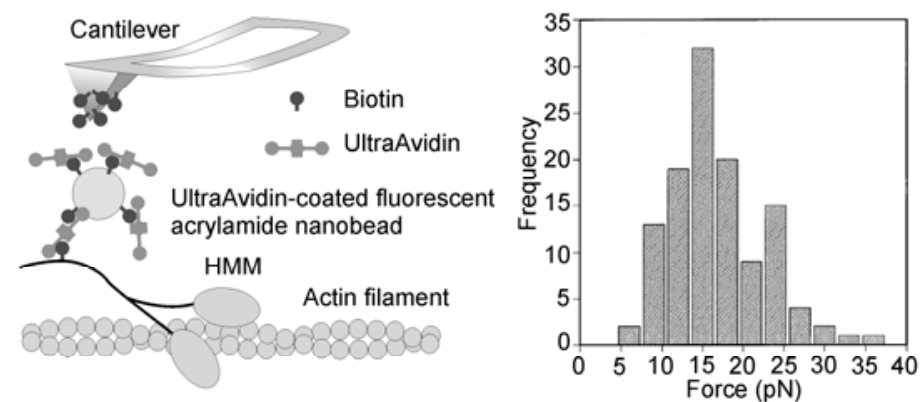

(b)

Figure 2 (a) Measurement of force generated by myosin motor on thin filament [21]; (b) measurement of interaction force during the binding process [31]. 
Combined with the latest researches on molecular mechanics and single molecule experiments, we analyzed the interactions between myosin motor and actin filament during their binding process, aiming at the working cycle of myosin motors [32,33]. The results revealed the operation rules of myosin motors under the coupling effects of van der Waals force, Casimir force and electrostatic force, and established the microscopic dynamical model based on the protein-protein interaction potential. Monte Carlo simulations of the dynamical model were further carried out, and comparing the influence of these forces, we found that electrostatic force plays a dominant role during the binding process, consistent with corresponding experimental measurements. Our work may facilitate further researches on the working cycles of molecular motors because it provided the mechanical rules of microscopic environment for molecular motors.

\subsection{Working mechanism of molecular motors}

(i) The cyclic working mechanism of a single motor. There are two mainstream-models for the cycling mechanism of a single molecular motor $[34,35]$. The first one is the conformational change model, which is relatively deterministic, and it attributes the force and displacement of the motor to the internal rearrangement and deformation of its molecular groups. The other one is the biased Brownian ratchet model, which is stochastic description based on statistical thermodynamics. It regards the motion of molecular motors as biased Brownian motion in a ratchet-shaped periodic potential. Each of these two models contributes to the research of molecular motors with some successes, while both have their own limitations and contradictions remain between them.

(1) The conformational change model. The earliest widely accepted conformational change model, which is called the cross-bridge model, was proposed by Huxley [35]. The cross-bridge refers to the structure formed by the strong binding between motor head and actin filament. Huxley suggested that such structures reinforce the stiffness of sarcomere, and the motor force as well as the relative sliding between thick and thin filaments comes from the rotation of motor head. The cross-bridge model provided pioneering theoretical explanations to the experimental phenomena at that time, while it laid significant foundations for the research, and the model is not sufficiently elaborate. Thus it can only be regarded as the qualitative explanation for the working mechanism of molecular motors. Single molecule experiments have been promoting the studies: The experiments conducted by Kaya et al. [36] indicated that myosin moves $\sim 8 \mathrm{~nm}$ during one power stroke, and the nonlinear elasticity of the neck region pronouncedly affects the force generation of the motor. Sellers et al. [37] directly observed the reversibility of the motor conformation during the power stroke via optical trap technology. On the other hand, based on the preceding researches, Spudich proposed the swinging cross-bridge model [26] or the lever arm model [38], which refined and improved the cross-bridge model. The lever arm model not only investigated the critical functions of several loops within motor head for its binding with actin and ATP, but also confirmed that the location of the motor domain is in the vicinity of the joint between motor head and neck region. As shown in Figure 3(a), the lever arm refers to the motor neck, and after the hydrolysis of ATP, the molecular groups within the motor domain go through rearrangement and deformation, which is amplified by the neck region. The linear displacement is produced by the $\sim 70^{\circ}$ rotation of the neck, whose elementary light chain (ELC) and regulatory light chain (RLC) regulate the stiffness of the lever arm. The lever arm model, which is still effective at present, is recognized as the second conformational change model, and it has been serving as a blue print for more detailed experimental researches after then. It is worth noting that the Osaka group in Japan has made great contributions to the single molecule experiments of molecular motors. They simultaneously observed ATP hydrolysis events and the dynamic variation of the displacement as well as system rigidity for the first time [39], by means of combining optical tweezers technology and fluorescent microscopy, as shown in Figure 4. Besides, they revealed the significant influence of the spatial angle between myosin head and actin filament on the displacement measurement [40], and observed the details of myosin movements via micro-tips. The experimental principles are shown in Figure 5(a), and (a)

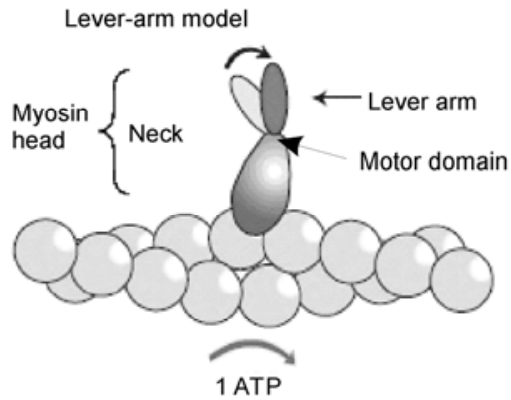

(b)

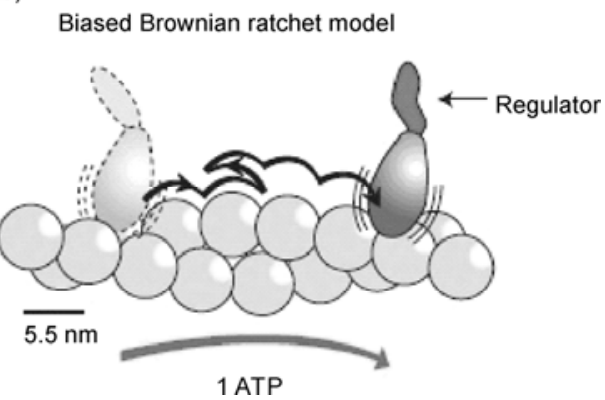

Figure 3 (a) The conformational change model; (b) the biased Brownian ratchet model [34]. 


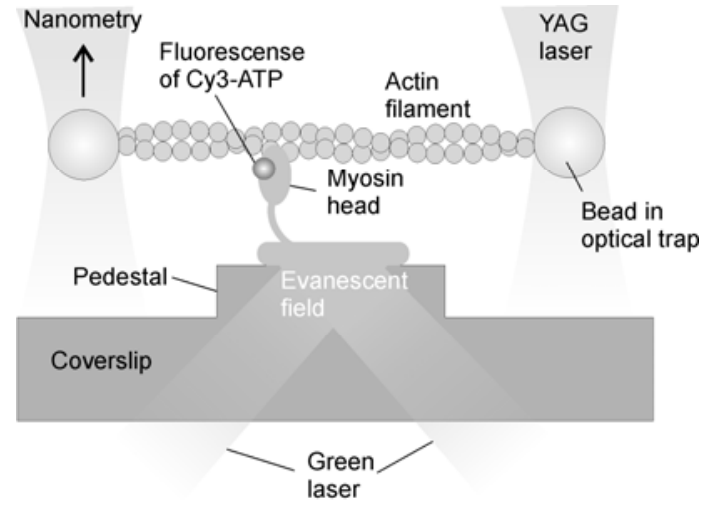

Figure 4 The apparatus of fluorescent single molecule experiment for molecular motors.

the results showed that when the motor head was in the optimal direction, it was able to move $\sim 15 \mathrm{~nm}$ during one ATP hydrolysis cycle, while the whole process was composed of several substeps, each of which is $\sim 5.5 \mathrm{~nm}$, as shown in Figure 5(b). Another fact they discovered is that the displacement of molecular motors can occur several milliseconds after the ATP hydrolysis, i.e. the motor can restore ATP energy, while the binding with actin starts its working cycle.

The interactions among molecular groups or single molecules form the physical origin of the conformational change model, which involves quantum mechanics (QM) and molecular mechanics (MM). Researches aimed at the model are based on single molecule experiments or MDS, with the purpose of determining what changes of which molecular groups within myosin result in the force generation and deformation of the motor. Recently, a QM/MM hybrid method has been applied in occasions like the enzymatic reactions of biological large molecules. This method calculates interactions at atomic level with QM, and those of molecular level with MM, and thereby such combinations can achieve the optimal balance between calculation cost and accuracy. With the progress of the QM/MM hybrid method, this highly efficient tool has succeeded in researches of the structures or reactions of biological large molecules [41-43], and provided new ideas for further

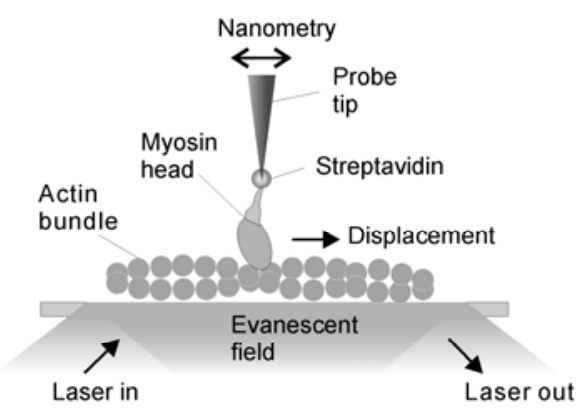

(a) studies of the working mechanism of molecular motors.

(2) The Brownian ratchet model. The conception of Brownian ratchet was firstly proposed by Feynman [44], who assumed an imaginary micro-ratchet, which makes it easier for particles to diffuse in one direction (ramp potential), but harder to diffuse in the opposite way (steep potential). Thus the probability for particles to diffuse in some direction would be higher, leading to the macroscopic unidirectional transport of mass. In real physical applications, such imaginary ratchets are equivalent to biased potential distribution, which is called ratchet potential. According to the description of the Brownian ratchet model for molecular motors, the myosin head can be regarded as a Brownian particle diffusing in cytoplasm, while the periodic ratchet potential originates from the periodic myosin binding sites on the thin filament. As for the reason why the potential is biased, different opinions exist, such as due to the anisotropic structure of myosin head or the binding of ATP [45]. The Brownian ratchet model attributes the force and displacement of the motor to its forward surfing under the effect of ratchet potential. In spite of occasional backward motions, the resultant motion is forward from the sense of statistical mean, as shown in Figure 3(b). The essential mechanism of the model is described by Langevin equation, in which the inertia term is generally neglected due to the tiny mass of the motor moving in the over-damped solution. The detailed descriptions of the model can be classified into the following forms [46]: fluctuating force, fluctuating potential and fluctuation between states. When the state of the motor is near equilibrium, the connection between energy transformation and particle transport can be expressed by Onsager relation, and at least one of the transitions among the states should break the detailed balance for the occurrence of unidirectional transport. Esaki et al. [47] made simulations for the Brownian motion of molecular motors in order to explain the data of single molecule experiments. Domestically, Ai et al. [48] established a physical stochastic inclined rod model to study the directional movement of myosin. Bao et al. [49] proposed a biased fluctuation model with a colored noise to study the stepping motion of molecular motors, and simulated the force-velocity relation via

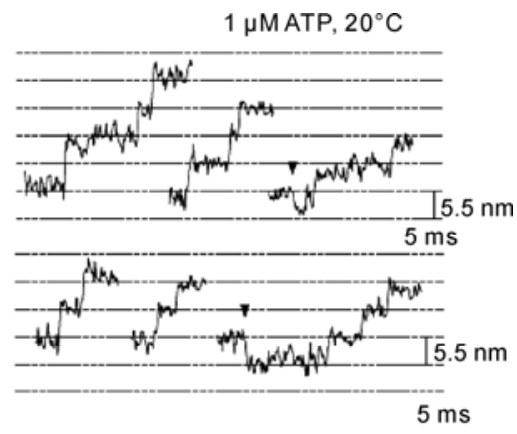

(b)

Figure 5 (a) The apparatus for displacement measurement of a single molecular motor during one power stroke; (b) the substeps of a single motor during one power stroke [34]. 
Monte-Carlo method. Li et al. [50] took research on the directional motion of myosin VI with a dipole model.

As shown in Figure 5(b), the experimental data showed that by consuming one ATP, a motor can move $\sim 15 \mathrm{~nm}$, which consists of several substeps of $\sim 5.5 \mathrm{~nm}$ [34]. The Osaka group who completed the experiment suggests that the size of the substep coincides with the interval between actin monomers (G-actin), and therefore they speculated that the motion of molecular motors results from Brownian ratchet mechanism. However, if a motor can repeatedly detach from the actin filament during one ATP hydrolysis cycle, it is hardly convincing that the motor is able to work effectively. On the other hand, the motor did not detach from the thin filament referring to Figure 5(b), in which all the substeps are continuous, so the consistence between the $5.5 \mathrm{~nm}$ substep and G-actin intervals is perhaps a coincidence, while for the power stroke of the motor, the conformational model seems more reasonable. This means that the substeps may result from the stepping release of ATP energy, indicating that there exist 4-5 energy levels inside of myosin molecular structure, and the transitions among the levels need to be analyzed with QM/MM method from the perspective of free energy. Thus, when applying the Brownian ratchet model, the researchers over focused on its functionality but neglected some fundamental problems, i.e. the physical meaning of the model should be matched and unified with the object considered (molecular motors).

The comparison between the conformational change model and the Brownian ratchet model is shown in Table 1. The conformational change model owns supports from experimental data although it needs further improvement, and for situations where myosin detaches from actin during post rigor state, the effect of ATP hydrolysis on molecular group structures during pre-power stroke and the force/displacement generation during power stroke, the model provides direct and effective descriptions. However, the model cannot explain why molecular motors are capable of moving 15 $\mathrm{nm}$ in one ATP hydrolysis cycle (existent models predict a displacement of 5-10 nm) and how the substeps are formed. Especially for the pre-power stroke, the model does not give reasonable explanation of how the motor selects next binding site and approaches the actin filament. Recently, Spudich found the experimental results of myosin VI quite challenging to the swinging cross-bridge model, because its moving distance exceeded that predicted by the model [51].
The research conducted by Sweeney et al. [52] on myosin VI indicated that the working rules of molecular motors need to be rewritten. Consequently, it is likely that not only the conformational change mechanism takes effect during the working of motors. The Brownian ratchet model has advantages on describing the process from myosin detaching from actin to its rebinding, and the theoretical results can indeed coincide with experimental data through proper adjustment, while its limitation is brought about by the fact that it lays too much emphasis on the mathematical descriptions and the over abstract interpretations. The model itself is quite functional, leaving the physical source of the potentials and forces ambiguous, including the tilt reasons of the energy landscape. Moreover, the Brownian ratchet model neglects the molecular structures of the acto-myosin system to a large extent, and many parameters of the model need manual adjustments so as to explain the experimental phenomena. All these aspects have weakened the effectiveness of the model. We see that the origins of the conformational change model and the Brownian ratchet model are respectively QM/MM and statistical thermodynamics, so their mechanical foundations are inconsistent with each other, and both advantages and limitations exist in these two models. Thus, for the working mechanism research of molecular motors, the key point is to unify the existent models, i.e. to find unified descriptions for QM, MM and statistical thermodynamics involved in motor motion.

For the reason that biological molecular motors are naturally optimized molecular machines, the researches on their working mechanism have also promoted the technologies of artificial molecular motors and nanomanufacturing, and the design and manufacture of biological nano-parts used as medicine carriers with molecular assembly have become the global frontier of biological nanotechnology research. The group of Montemagno [53] made a rotary molecular motor, which was based on ATPase motors, and a simple micro screw propeller was obtained via grafting a nickel rod to the ATPase central axis. Van Delden et al. [54] made an optical driving molecular motor based on the working mechanism of ATPase motors. Zhao et al. [55] constructed a nano propeller by connecting a nickel nano-wire to the $\mathrm{F}_{1}$-ATPase motor, and realized its rotation control by designing a regulatory magnetic field. Cui et al. [56] observed the rotation of an optical driving $\mathrm{F}_{0} \mathrm{~F}_{1}$-ATPase composite. Qi et al. [57] assembled ATPase motors extracted from plants to micro

Table 1 The comparison between the conformational change model and the Brownian ratchet model

\begin{tabular}{lll}
\hline Characteristics & Conformational change model & Brownian ratchet model \\
\hline Mechanical foundation & QM/MM & Statistical thermodynamics \\
Description manner & Relatively deterministic & Relatively stochastic \\
Analysis method & MDS & Physical modeling/stochastic simulation \\
Relation with the structures of biological molecules & Close & Not close \\
Application scope & Binding/detaching between myosin & The motion of myosin after detaching; \\
& and actin; the power stroke & selection of binding sites \\
\hline
\end{tabular}


capsules modified by phosphorus. With the discussion above, we see that the working mechanism research of molecular motors holds important theoretical significance.

(ii) Collective working mechanism of molecular motors. For the reason that myosin II is non-processive linear motor with low duty ratio and its dwell time on the thin filament is very short, therefore in order to generate macroscopic force and movement, the collective work satisfying statistical rules of huge amounts of motors is essential. It is an effective method to build a new biomechanical model from microscopic to macroscopic level based on the collective behavior of myosin motors with statistical mechanics. Current studies on the collective working mechanism for myosin motors are mainly focused on two aspects. From the perspective of chemical kinetics, Lan et al. [58] presented a mechanical model for steady muscle contraction based on the conformational changes of a single motor, and studied the force-velocity relation of contraction, taking the actin filament as the object. Chin et al. [59] described the cycling process of molecular motors with a 7-state model and further modified the force-velocity relationship. Guo et al. [60] provided a mechanochemical coupling model for the working cycle of myosin, and investigated the spontaneous vibration of muscle by describing the collective behavior of myosin motors with a set of chemical dynamical equations. Starting from statistical physics, Julicher et al. [46] analyzed the collective characteristics of molecular motors via proposing a two-state ratchet model based on statistical mechanics, and established corresponding statistical equations. Based on the same model, Shu et al. [61] discussed the effects of collective features on the hydrolysis dynamics of ATP. The actual mechanical properties of the system are not easy to present with physical methods since they are mainly aimed at qualitative analyses of motor dynamics. Chemical methods analyze the contraction mechanism based on the chemical states of the motor, while it lacks the unified mathematical description of the contraction process and the quantitative calculation of the collective features of molecular motors.

Further researches on the collective cooperation mechanism of myosin motors are needed. A new mathematical model should be established by combining the merits of chemical kinetics with statistical physics, and the factors affecting the collective properties such as the [ATP] in sarcoplasm [62], the external load on molecular motors [63] and the interaction potentials between motors can be discussed. Brugues et al. [64] analyzed the collective dynamics of molecular motors under the external load and considered the effects of interaction potentials. The calculation results showed that unequal loading enhanced cooperativity, leading to higher efficiencies than those of single motors. Campas et al. [65] studied the collective behaviors of $N$ molecular motors and found that the interactions between motors determine the force-velocity curves, which differ significantly from those of single motors. We see that for the col- lective working process, the effects of load must be taken into account. We analyzed the collective behaviors of myosin with non-equilibrium statistical mechanics and established the Fokker-Planck equation containing the relative displacement between myosin and actin filament under load according to the geometric structure of sarcomere [66]. We further deduced the mechanical model of sarcomere contraction, and investigated the effects of [ATP] and loads on the active force and velocity of muscle contraction based on the coupling relation between [ATP] and the transition rates between motor states. The results showed that under certain number of motors, cross-section area and elasticity coefficient, muscle active force gained with the rise of [ATP], and the contraction speed gradually declined to zero with the increase of external load, while the contraction speed of sarcomere was positively correlated to [ATP] and stayed constant when [ATP] is saturated.

According to the microscopic process of muscle contraction, the working of molecular motors is regulated by $\mathrm{Ca}^{2+}$ due to the conformational changes of Tm induced by the binding of $\mathrm{Ca}^{2+}$ to Tn. Alencar et al. [67] analyzed the contraction mechanism of muscle from this perspective. Stein et al. [68] studied the relation between $\left[\mathrm{Ca}^{2+}\right]$ and muscle force. Considering the activation of the thin filament by the binding of $\mathrm{Ca}^{2+}$, the myoplasmic $\left[\mathrm{Ca}^{2+}\right]$ deciding the number of working motors can be employed to characterize the activation degree of the actin filament. Thus the influence of $\left[\mathrm{Ca}^{2+}\right]$ on the collective behavior of myosin motors can be discussed based on the kinetics of the binding process between $\mathrm{Ca}^{2+}$ and $\mathrm{Tn}$. On the other hand, myoplasmic $\left[\mathrm{Ca}^{2+}\right]$ is determined by the features of AP viewing from the excitation process of skeletal muscle. Therefore, another important aspect of the transition from microscopic to macroscopic level for the research of biomechanical models is to investigate the relations between AP firing rate and $\left[\mathrm{Ca}^{2+}\right]$, active force and contraction velocity from the perspective of cybernetics. We analyzed corresponding problems in [69] and established a dynamical model for skeletal muscle contraction based on the serial and parallel features of sacromeres.

\section{Bioelectrochemical driving and control mechanisms of skeletal muscle}

\subsection{Bioelectrochemical driving mechanisms of skeletal muscle}

The driving mechanism research of skeletal muscle is focused on how motor neurons stimulate muscle fibers and further start the working cycles of molecular motors, and how the contraction of muscle fibers, as a bioelectrochemical process, is regulated. When the APs of motor neurons arrive at the neuromuscular junction, they will be regenerated by the sarcolemma [70]. Thus the APs on sarcolemma and motor neurons reflect the motion intention of human 
body and contain control information of skeletal muscle. The essential condition for molecular motors to work is that $\mathrm{Ca}^{2+}$ binds with $\mathrm{Tn}$ on the thin filament. Under normal operation of sarcomeres, the myoplasmic [ATP] is almost saturated, while $\left[\mathrm{Ca}^{2+}\right]$ determines the operation probability of the motors, and therefore $\mathrm{Ca}^{2+}$ activities indicate the driving information of molecular motors.

The biological process of skeletal muscle contraction is shown in Figure 6(a). APs propagate along T-tubules, leading to the release of $\mathrm{Ca}^{2+}$ in the SR into cytoplasm, then $\mathrm{Ca}^{2+}$ binds with $\mathrm{Tn}$, finally molecular motors start to work and sarcomeres begin to contract. The whole process is referred to as the excitation-contraction coupling (ECC) [4]. myoplasmic $\mathrm{Ca}^{2+}$ is recycled by Ca-ATPase on SR [71], therefore the $\left[\mathrm{Ca}^{2+}\right]$ of sarcomeres is always at dynamic equilibrium, while during the normal work of muscle fibers, the $\mathrm{Ca}^{2+}$ channels and Ca-ATPase on SR are dominating. As previously discussed, the excitation degree and the power of skeletal muscle are characterized by the number of working molecular motors, so the excitation degree is also represented by myoplasmic $\left[\mathrm{Ca}^{2+}\right]$. For the driving mechanism research of skeletal muscle, it is essential to further explore the mechanism of ECC and make corresponding theoretical models and descriptions. Yin et al. [72] found that there exist physical couplings among the $\mathrm{Ca}^{2+}$ channels on SR, i.e. the depolarization or repolarization of the membrane can result in rapid open or close of these channels, and thus AP has been verified to be the regulation and control source of $\left[\mathrm{Ca}^{2+}\right]$. Stern et al. [73] built physical models of ECC in sarcomeres based on the physiological structures of sarcolemma. Cannell et al. [74] simulated the movement of $\mathrm{Ca}^{2+}$ in sarcomeres of frog, while the model neglected the detailed physiological properties of sarcolemma. Stuyvers et al. [75] investigated the steady relationship between $\left[\mathrm{Ca}^{2+}\right]$ and firing rate of AP.

Current studies on the driving mechanism of sarcomeres are mostly aimed at the static features of myoplasmic $\left[\mathrm{Ca}^{2+}\right]$ or the steady relation between $\left[\mathrm{Ca}^{2+}\right]$ and firing frequency, while the dynamic variation and spread of $\left[\mathrm{Ca}^{2+}\right]$ or its dy- namic regulation by AP are seldom covered. On the other hand, experiments have shown that the firing rate of sarcolemma AP is positively correlated to the isometric tension of muscle [76], indicating that similar relations exist between the firing rate and $\left[\mathrm{Ca}^{2+}\right]$. In other words, $\left[\mathrm{Ca}^{2+}\right]$ is regulated by the AP firing rate. In [77], we attempted to make progress against existing problems via establishing cybernetic models of the formation of AP on sarcolemma and how it dynamically regulates the $\left[\mathrm{Ca}^{2+}\right]$ in sarcomeres. Combined with our corresponding experiments, we proposed the bioelectrochemical control mechanism with variable-frequency regulation for skeletal muscle. At present, however, we only obtained the open-loop model of muscle contraction, without taking into account the feedback effect of proprioceptors. Open-loop systems have difficulties in representing the real motion intention due to its insufficient accuracy, and thus it is essential to investigate the closedloop control mechanism of skeletal muscle.

\subsection{Control mechanisms of skeletal muscle}

From the perspective of cybernetics, the contraction of skeletal muscle is a typical closed-loop control process. The movements of human body are controlled by the neural systems. As shown in Figure 6(b), neural signal propagates from the central nervous system (CNS) to the corticospinal tract in the form of AP, and arrives at motor neurons to control the contraction of muscle fibers, thereby AP is the information carrier of motion intention, and the control information of molecular motors is implied in AP produced by motor neurons. The first complete mathematical description of AP was provided by Hodgkin and Huxley [78], who modeled the $\mathrm{Na}^{+}$current, $\mathrm{K}^{+}$current and the leakage current across the membrane and summarized the electrical model of membrane via voltage-clamp experiments, in which squid giant axons were taken as the experimental objects. With the development of computing technology, more refined models have been proposed since then. Based on existent abstract models, Luscher et al. [79,80] investigated

(a)

Motor

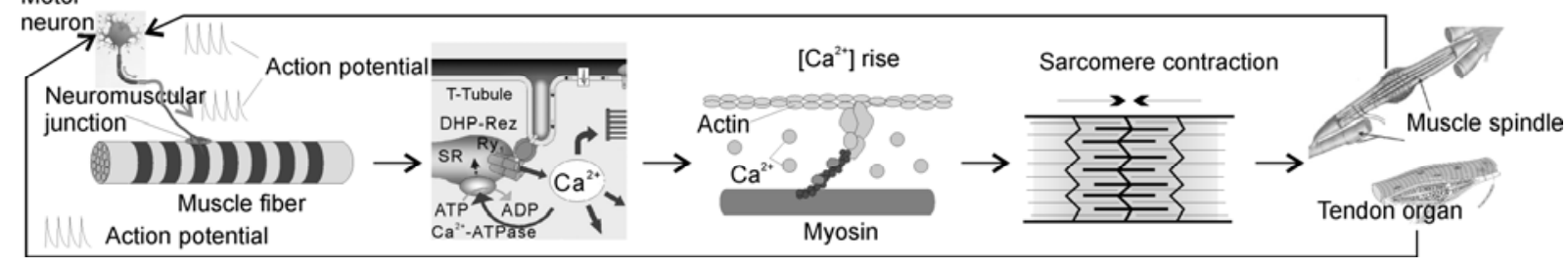

(b)

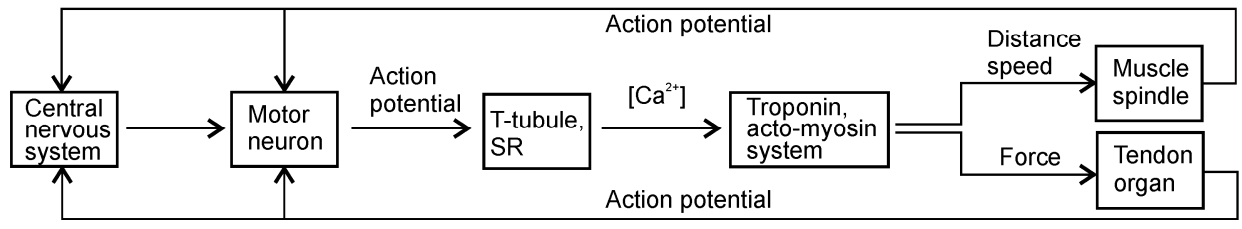

Figure 6 (a) The biological process of skeletal muscle contraction; (b) the control diagram of muscle contraction. 
the influence of bifurcations in neuron structures on AP propagation features, as well as the effect of axon geometric size on the conduction speed and frequency of AP. Rogers et al. [81] modeled and simulated the propagation features of cardiac AP with finite element method, and studied the decelerating effect of inhomogeneous and anisotropic structures of muscle fibers on AP conduction, including the unidirectional blocking effect produced by the curvature of AP wave front.

As shown in Figure 6(b), the proprioception of motion is fed back to the CNS and motor neurons via AP. The hardware basis for the inspection of motion information is proprioceptor, which transmits information to the CNS via sensing the speed, tension and stretch length of muscle, so as to realize its harmonious control. The proprioceptors of muscle mainly include muscle spindle and tendon organ (Figure 6(a)), which are equivalent to the displacement/ speed sensor and tension sensor, respectively. For the motor neuron of a motor unit, tendon organ gives negative feedback signal, i.e. it generally inhibits muscle contraction, while muscle spindle transmits positive signal that potentiates the contraction.

With the above discussion, we see that biological neural networks are information feedback loops and the central control unit [82], so it is important to investigate how motor neurons receive synaptic feedback inputs and how they process and regulate the output. Relevant researches have shown that the distribution density and geometric patterns of ion channels influence the amplitude, conduction speed and firing rate of AP, and these physical quantities are coupled to each other. Furthermore, due to the "all or none" property of AP, the firing rate is critical to the control of molecular motors, thus we need to identify what factors/ physical quantities have effects on the firing rate, and study the feedback and fusion mechanism of AP frequency information. This involves the problem of how motor neurons modulate the AP fed back by muscle spindle and tendon organ and make the system stable, facilitating the dynamic regulation of muscle contraction. On the other hand, at present little is known about how biological neural networks achieve adaptive learning according to the feedback of proprioceptors, and consequently it remains difficult to build bionic adaptive motion controllers, which we believe to be a key point of control mechanism research of skeletal muscle in the near future.

\section{Conclusions and prospect}

Based on the above discussion, we know that the biomechanical research of skeletal muscle is aimed at the microscopic mechanism of muscle contraction, the driving and control mechanism and the modeling of corresponding mechanics and control systems, in order to further explore the applications of the models in subjects like bionics and bio- mechanics. Focusing on the existent difficulties, the following aspects need to be further studied.

(1) The working mechanism and mechanical principium of molecular motors. The dynamic behavior and the working mechanism of molecular motors under multi-force coupling interactions during the cycles should be investigated. The dynamical characteristics of myosin within an ATP hydrolysis cycle should be analyzed with QM/MM hybrid method, and a more advanced new theory of single-motor working mechanism needs to be established with the combination of the conformational change model and the Brownian ratchet model.

(2) The experimental analyses on the mechanical features of molecular motors. In order to investigate the working rules of motors, experimental researches are essential. With the help of pico-Newton level devices such as AFM and optical tweezers, the interactions between myosin and actin filaments can be measured by elaborate design of experiments, so as to explore the multi-force coupling and the working mechanisms of molecular motors.

(3) The collective working mechanism of molecular motors. The mechanochemical coupling characteristics of molecular motors can be analyzed via employing non-equilibrium statistical mechanics, and the dynamical equations of the acto-myosin system should be established. The collective mechanical properties of molecular motors need to be verified by novel micro force detection apparatus at the level of sarcomere or myofibril.

(4) The biomechanical model of skeletal muscle. The relations between AP firing rate and muscle tension or contraction speed need to be further studied, and the biomechanical model for skeletal muscle contraction can be established based on the collective behavior of molecular motors, aiming at the serial and parallel features of sarcomeres. Combining with the research strategies of the biomechanical model, the joint forces (torque) of human body and the physiological signal (sEMG) can be collected via macroscopic experiments, and the biomechanical model based on microscopic mechanics can be verified through the dynamical analysis of human body joints.

(5) The cybernetic model of skeletal muscle. The physical basis of muscle driving and control should be elucidated, i.e. how AP regulates the $\left[\mathrm{Ca}^{2+}\right]$ of muscle fibers, and corresponding physical models need to be built. If the cybernetic model of the driving process is obtained, the transfer functions from AP to $\left[\mathrm{Ca}^{2+}\right]$, and that from $\left[\mathrm{Ca}^{2+}\right]$ to muscle force can be completed. For the closed-loop control model of skeletal muscle, the fusion and stabilization mechanism of the contraction speed/force information fed back by muscle spindles and tendon organs needs to be studied, and the theory should be verified by corresponding experiments. The cybernetic model may provide guidance for the bionic design and control of artificial muscle.

The biomechanical research of skeletal muscle is full of challenges, due to its massive contents, broad concerns and 
large span. Despite of these difficulties, the research has attracted many scholars to make exploration for its theoretical significance and potential application value. With the continued introduction of new methods and technologies, it is convincing that more rules will be revealed, so that profound influences will be made on fields like the exploration of human body motion mechanism and rehabilitation engineering.

This work was supported by the National Natural Science Foundation of China (61075101, 60643002), the National Basic Research Program of China (2011CB013203), the Science and Technology Intercrossing and the Medical and Technology Intercrossing Research Foundation of Shanghai Jiao Tong University (LG2011ZD $D_{1} 06$, YG2010ZD $\left._{1} 01\right)$.

1 Hill A V. The heat of shortening and the dynamic constants of muscle. Proc R Soc Lond B, 1938, 126: 136-195

2 Huxley A F, Niedergerke R. Structural changes in muscle during contraction. Nature, 1954, 173: 971-973

3 Huxley H E, Hanson J. Changes in the cross-striations of muscle during contractions and stretch and their structural interpretation. Nature, 1954, 173: 973-976

4 Maclntosh B R, Gardiner P F, McComas A J. Skeletal Muscle: Form and Function. 2nd ed. Champaign, IL: Human Kinetics, 2005. $151-175$

5 Li Y S, Chen W Y. Constitutive models of skeletal muscle contraction: I passive behaviors (in Chinese). Adv Mech, 2010, 40: 663-678

6 Linke W A, Ivemeyer M, Mundel P, et al. Nature of PEVK-titin elasticity in skeletal muscle. Proc Natl Acad Sci USA, 1998, 95: 8052-8057

7 Rayment I, Holden H M, Whittaker M. Structure of the actin-myosin complex and its implications for muscle contraction. Science, 1993, 261: 56-65

8 Rayment I, Rypniewski W R, Schmidt-Base K, et al. Three dimensional structure of myosin subfragment-1: A molecular motor. Science, 1993, 261: 50-58

9 Uyeda T Q, Abramson P D, Spudich J A. The neck region of the myosin motor domain acts as a lever arm to generate movement. Proc Natl Acad Sci USA, 1996, 93: 4459-4464

10 Holmes K C, Angert I, Jon K F, et al. Electron cryo-microscopy shows how strong binding of myosin to actin releases nucleotide. Nature, 2003, 425: 423-427

11 Fung Y C. Biomechanics: Mechanical Properties of Living Tissues. New York: Springer-Verlag, 1993. 568

12 Li Y S, Zhang Y Q, Chen W Y. The constitute model of skeletal muscle contraction (in Chinese). J Taiyuan Univ Tech, 2005, 36: 760-764

13 Zajac F E. Muscle and tendon: Properties, models, scaling, and application to biomechanics and motor control. Crit Rev Biomed Eng, 1989, 17: 359-411

14 Huxley H E. The mechanism of muscular contraction. Science, 1969, 164: 1356-1366

15 Huxley A F. Muscle structure and theories of contraction. Prog Biophys Biophys Chem, 1957, 7: 255-318

16 Huxley A F, Simmons R M. Proposed mechanism of force generation in striated muscle. Nature, 1971, 233: 533-538

17 Gordon A M, Huxley A F, Julian F J. The variation in isometric tension with sarcomere length in vertebrate muscle fibres. J Physiol, 1966, 184: 170-192

18 Zahalak G I, Motabarzadeh I. A re-examination of calcium activation in Huxley cross-bridge model. J Biomech Eng, 1997, 119: 20-29

19 Piazzesi G, Reconditi M, Linari M, et al. Mechanism of force generation by myosin heads in skeletal muscle. Nature, 2002, 415: 659-662

20 Finer J T, Simmons R M, Spudich J A. Single myosin molecule mechanics: Pico Newton forces and nano metre steps. Nature, 1994, 368: 113-119
21 Anderson F C, Pandy M G. Static and dynamic optimization solutions for gait are practically equivalent. J Biomech, 2001, 34: 153161

22 Neptune R R, Burnfield J M, Mulroy S J. The neuromuscular demands of toe walking: A forward dynamics simulation analysis. $\mathrm{J}$ Biomech, 2007, 40: 1293-1300

23 Yang Y Y, Wang R C, Wang Y L, et al. Forward dynamics analysis of human lower limb neuromusculoskeletal system (in Chinese). J Tsinghua Univ (Sci Tech), 2006, 46: 1872-1875

24 Shu Y G, Ouyang Z C. Biological molecular motors (in Chinese). Physics, 2007, 36: 735-741

25 Yamakita Y, Iio T. Conformational change of skeletal muscle troponin. J Biochem, 1989, 105: 870-874

26 Spudich J A. The myosin swinging cross-bridge model. Nat Rev Mol Cell Biol, 2001, 2: 387-392

27 Lymn R W, Taylor E W. Mechanism of adenosine triphosphate hydrolysis by actomyosin. Biochemistry, 1971, 10: 4617-4624

28 Zhao Y P. Some physical mechanical problems in Nano-electromechanical Systems (in Chinese). Chin Mech Abst, 2007, 21: 1-21

29 Munday J N, Capasso F, Parsegian V A. Measured long-range repulsive Casimir-Lifshitz forces. Nature, 2009, 457: 170-173

30 Liu Y M, Scolari M, Im W, et al. Protein-protein interactions in actinmyosin binding and structural effects of R405Q mutation: A molecular dynamics study. Proteins: Struct Funct Bioinform, 2006, 64: 156-166

31 Nakajima H, Kunioka Y, Nakano K, et al. Scanning force microscopy of the interaction events between a single molecule of heavy meromyosin and actin. Biochem Biophys Res Commun, 1997, 234: 178182

32 Guo Z, Yin Y H. Coupling mechanism of multi-force interactions in the myosin molecular motor. Chin Sci Bull, 2010, 55: 3538-3544

33 Guo Z, Yin Y H. Casimir effect on adhesion interaction between myosin molecular motor and actin filament. Inter J Nanosyst, 2010, 3: 9-15

34 Yanagida S, Kitamura K, Tanaka H, et al. Single molecule analysis of the actomyosin motor. Curr Opin Cell Biol, 2000, 12: 20-25

35 Huxley A F. Cross-bridge action: Present views, prospects, and unknowns. J Biomech, 2000, 33: 1189-1195

36 Kaya M, Higuchi H. Nonlinear elasticity and an 8-nm working stroke of single myosin molecules in myofilaments. Science, 2010, 329: 686-689

37 Sellers J R, Veigel C. Direct observation of the myosin-Va power stroke and its reversal. Nat Struct Mol Biol, 2010, 17: 590-595

38 Uyeda T Q P, Abramson P D, Spudich J A. The neck region of the myosin motor domain acts as a lever arm to generate movement. Proc Natl Acad Sci USA, 1996, 93: 4459-4464

39 Ishijima A, Kojima H, Funatsu T, et al. Simultaneous observation of individual ATPase and mechanical events by a single myosin molecule during interaction with actin. Cell, 1998, 92: 161-171

40 Yanagida T, Iwaki M, Ishii Y. Single molecule measurements and molecular motors. Phil Trans R Soc B, 2008, 363: 2123-2134

41 Li G H, Cui Q. Mechanochemical coupling in myosin: A theoretical analysis with molecular dynamics and combined QM/MM reaction path calculations. J Phys Chem B, 2004, 108: 3342-3357

42 Yang Z, Zhao Y P. QM/MM and classical molecular dynamics simulation of His-tagged peptide immobilization on nickel surface. Mat Sci Eng A-Struct, 2006, 423: 84-91

43 Yang Z, Zhao Y P. Adsorption of His-tagged peptide to $\mathrm{Ni}, \mathrm{Cu}$ and $\mathrm{Au}$ (100) surfaces: Molecular dynamics simulation. Eng Anal Bound Elem, 2007, 31: 402-409

44 Feynman R P, Leighton R B, Sands M. The Feynman Lectures on Physics. Boston: Addison-Wesley Longman, 1970

45 Astumian R D. Thermodynamics and kinetics of a Brownian motor. Science, 1997, 276: 917-922

46 Julicher F, Ajdari A, Prost J. Modeling molecular motors. Rev Mod Phys, 1997, 69: 1269-1281

47 Esaki S, Ishii Y, Yanagida T. Model describing the biased Brownian movement of myosin. Proc Japan Acad, 2003, 79: 9-14

48 Ai B Q, Wang X J, Liu G T, et al. Theoretical study for muscle contraction (in Chinese). Chin J Med Phys, 2003, 20: 107-109 
49 Bao J D, Zhou Y Z. Biased fluctuation model for the unidirectional stepping motion of molecular motor (in Chinese). Chin Sci Bull (Chin Ver), 1998, 43: 1493-1496

50 Li C P, Hang Y R, Zhan Y, et al. Study the directional motion of myosin VI with the dipole model (in Chinese). Chin Sci Bull (Chin Ver), 2008, 53: 528-532

51 Spudich J A, Sivaramakrishnan S. Myosin VI: An innovative motor that challenged the swinging lever arm hypothesis. Nat Rev Mol Cell Biol, 2010, 11: 128-137

52 Sweeney H L, Houdusse A. Myosin VI rewrites the rules for myosin motors. Cell, 2010, 141: 573-582

53 Montemagno C, Bachand G. Constructing nanomechanical devices powered by biomolecular motors. Nanotechnology, 1999, 10: 225-231

54 Van Delden R A, Ter Wiel M K J, Pollard M M, et al. Unidirectional molecular motor on a gold surface. Nature, 2005, 437: 1337-1340

55 Ren Q, Zhao Y P, Yuek J C, et al. Biological application of multicomponent nanowires in hybrid devices powered by F1-ATPase motors. Biomed Microdevices, 2006, 8: 201-208

56 Cui Y B, Zhang Y H, Yue J C, et al. Direct observation of the clockwise light-driven rotation of $\mathrm{F}_{0} \mathrm{~F}_{1}$-ATP synthase complex. Chin Sci Bull, 2009, 49: 1235-1237

57 Qi W, Duan L, Wang K, et al. Motor protein $\mathrm{CF}_{0} \mathrm{~F}_{1}$ reconstituted in lipid-coated hemoglobin microcapsules for ATP synthesis. Adv Mater, 2008, 20: 601-605

58 Lan G, Sun S X. Dynamics of myosin-driven skeletal muscle contraction I. Steady-state force generation. Biophys J, 2005, 88: 41074117

59 Chin L, Yue P, Feng J J, et al. Mathematical simulation of muscle cross-bridge cycle and force-velocity relationship. Biophys J, 2006, 91: 3653-3663

60 Guo W S, Luo L F. A new model of the mechanochemical actin activated myosin ATPase cycle (in Chinese). Prog Biochem Biophys, 2003, 30: 216-220

61 Shu Y G, Shi H L. Cooperative effects on the kinetics of ATP hydrolysis in collective molecular motors. Phys Rev E, 2004, 69: 021912

62 Vermeulen K C, Stienen G J M, Schmid C F, et al. Cooperative behavior of molecular motors. J Muscle Res Cell Mot, 2002, 23: 71-79

63 Veigel C, Molloy J E. Load-dependent kinetics of force production by smooth muscle myosin measured with optical tweezers. Nat Cell Biol, 2003, 5: 980-986

64 Brugues J, Casademunt J. Self-organization and cooperativity of weakly coupled molecular motors under unequal loading. Phys Rev Lett, 2009, 102: 118104

65 Campas O, Kafri Y, Zeldovich K B, et al. Collective dynamics of interacting molecular motors. Phys Rev Lett, 2006, 97: 038101
66 Yin Y H, Guo Z. Collective mechanism of molecular motors and a dynamic mechanical model for sarcomere. Sci China Tech Sci, 2011, 54: 2130-2137

67 Alencar A M, Butler J P, Mijailovich S M. Thermodynamic origin of cooperativity in acto-myosin interactions: The coupling of shortrange interactions with actin bending stiffness in an Ising-like model. Phys Rev E, 2009, 79: 041906

68 Stein R B, Bobet J, Owuztoreli M N, el al. The kinetics relating calcium and force in skeletal muscle. Biophys J, 1988, 54: 705-717

69 Guo Z, Yin Y H. A dynamic model of skeletal muscle based on collective behavior of myosin motors-Biomechanics of skeletal muscle based on working mechanism of myosin motors (I). Sci China Tech Sci, 2012, 55: 1589-1595

70 Sanes J R, Lichtman J W. Development of the vertebrate neuromuscular junction. Ann Rev Neurosci, 1999, 22: 389-442

71 Toyoshima C, Nakasako M, Nomura H, et al. Crystal structure of the calcium pump of sarcoplasmic reticulum at $2.6 \AA$ resolution. Nature, 2000, 405: 647-655

72 Yin C C, D'Cruz L G, Lai F A. Ryanodine receptor arrays: Not just a pretty pattern? Cell, 2008, 18: 149-156

73 Stern M D, Pizzaro G, Rios E. Local control model of excitation-contraction coupling in skeletal muscle. J Gen Physiol, 1997, 110: 415-440

74 Cannel M B, Allen D G. Model of calcium movements during activation in the sarcomere of frog skeletal muscle. Biophys J, 1984, 45: 913-925

75 Stuyvers B D, McCulloch A D, Guo J, et al. Effect of stimulation rate, sarcomere length and $\mathrm{Ca}^{2+}$ on force generation by mouse cardiac muscle. J Physiol, 2002, 544: 817-830

76 Edwards R H T, Hill D K, Jones D A. Fatigue of long duration in human skeletal muscle after exercise. J Physiol, 1977, 272: 769-778

77 Yin Y H, Chen X. Bioelectrochemical control mechanism with variable-frequency regulation for skeletal muscle contraction-Biomechanics of skeletal muscle based on the working mechanism of myosin motors (II). Sci China Tech Sci, 2012, 55: 2115-2125

78 Hodgkin A L, Huxley A F. A quantitative description of membrane current and its application to conduction and excitation in nerve. $\mathbf{J}$ Physiol, 1952, 117: 500-544

79 Luscher H R, Shiner J S. Simulation of action potential propagation in complex terminal arborizations. Biophys J, 1990, 58: 1389-1399

80 Smith D O. Mechanisms of action potential propagation failure at sites of axon branching in the crayfish. J Physiol, 1980, 301: 243-259

81 Rogers J M, McCulloch A D. A collocation-Galerkin finite element model of cardiac action potential propagation. IEEE Trans Biomed Eng, 1994, 41: 743-757

82 Kandel E R, Schwartz J H, Jessell T M. Principles of Neural Science. 4th ed. New York: Elsevier, 2000

Open Access This article is distributed under the terms of the Creative Commons Attribution License which permits any use, distribution, and reproduction in any medium, provided the original author(s) and source are credited. 